\title{
Dietary Supplements and Sports Performance: Herbals
}

\author{
Melvin Williams \\ Department of Exercise Science, Old Dominion University, Norfolk, VA. Address correspondence to \\ mwilliam@odu.edu
}

Received April 17, 2006/Accepted April 20, 2006.

\section{ABSTRACT}

This is the fourth in a series of six articles to discuss the major classes of dietary supplements (vitamins; minerals; amino acids; herbs or botanicals; metabolites, constituents/extracts, or combinations). The major focus is on efficacy of such dietary supplements to enhance exercise or sport performance. Journal of the International Society of Sports Nutrition. 3(1):1-6, 2006

\section{Key Words: sports supplements, sports nutrition, herbs, botanicals, metabolites, extracts.}

\section{HERBALS: ERGOGENIC THEORY}

Plants provide us with most nutrients essential for life. Other than essential nutrients, plant foods contain naturally occurring substances, referred to respectively as phytochemicals. Herbals, which are derived from leaves, bark, berries, roots, gums, seeds, stems or flowers of plants, also contain numerous phytochemicals thought to have nutritive or medicinal value. Herbs have been used as medicine throughout history. Winslow and Kroll ${ }^{1}$ reported the earliest evidence of human use of plants for healing dates back to the Neanderthal period, and today various modern medicines may be classified as herbals. Thus, herbals are regulated as medicine in some countries, such as Germany, but as dietary supplements in others. Currently in the United States most herbals are regulated by the Dietary Supplement Health and Education Act (DSHEA), more like food ingredients than drugs. However, given the pharmacological effect of various herbals, some health professionals are emphasizing the need for regulations standardizing herbal therapy ${ }^{2}$.

Herbals are popular dietary supplements. In the most recent NHANES report, approximately 7 percent of the U. S. population takes herbal or botanical dietary supplements ${ }^{3}$. Athletes also take herbal supplements. For example, Herbold and others ${ }^{4}$ reported that 17 percent of female collegiate athletes used herbal/botanical supplements. Herbal dietary supplements are marketed to physically active individuals for a variety of reasons, including increasing energy, inducing weight loss, promoting muscle growth, or inducing other physiological or metabolic responses that may enhance exercise performance. For example, the product SportPharm contains multiple herbals, including Thermadrene, Ma Huang, Guarana, Caffeine, Purple Willow Bark, Cayenne pepper, and Ginger root, and is designed to increase mental alertness, stimulate fat-burning metabolism, and help enhance physical performance. Some sports drinks and sports bars contain herbals as well.

Research supports beneficial medicinal effects of specific herbs for specific health problems, as documented in Herbal Medicine: Expanded Commission E Monographs ${ }^{5}$ and WHO Monographs on Selected Medicinal Plants ${ }^{6}$. Unfortunately, however, with a few exceptions research investigating the ergogenic effects of herbal supplements is limited. The following discussion highlights available research with selected herbal products; other plant-derived substances, such as caffeine and ephedrine, will be discussed in a forthcoming issue.

\section{HERBAL SUPPLEMENTS: ERGOGENIC EFFICACY}

Capsaicin. The Capsicum species (C. annuum; $C$. frutescens), native to tropical America, incorporates such peppers as the cayenne, red, and chili. The medicinal properties of the capsicum species are attributable to a compound known as capsaicin ${ }^{7}$. 
The United States Pharmacopeia has classified capsaicin as a stimulant, and based on their previous research Lim and others ${ }^{8}$ have related its physiological action to caffeine, i.e., ingestion may induce sympathetic activation of the central nervous system, increasing catecholamine secretion and enhancing lipid oxidation, sparing the use of glycogen.

Some research supports this hypothesis. For example, when fed capsaicin $(2 \mathrm{mg} / \mathrm{kg})$, males at rest and exposed to immersion in cold water had a significant decrease in carbohydrate as an energy source ${ }^{9}$. However, limited research is available regarding the effect of capsaicin on carbohydrate metabolism during exercise. In a well-designed study, Lim and others ${ }^{8}$ evaluated the effect of a breakfast meal containing $10 \mathrm{~g}$ of dried, hot red pepper on energy substrate use in male runners during rest and exercise (cycling at about 60 percent $\mathrm{VO}_{2} \max$ ). For the red pepper trial, plasma epinephrine and norepinephrine concentrations were significantly elevated after 30 min, but not at 60 and $150 \mathrm{~min}$ of rest. The hot pepper meal significantly elevated the respiratory quotient (RQ) and blood lactate levels at rest and during exercise, but there was no effect on oxygen consumption or energy expenditure during rest or exercise. These results suggest that contrary to the theory of glycogen sparing, hot red pepper ingestion stimulates carbohydrate oxidation at rest and during exercise. The authors suggested that hot red pepper ingestion before exercise could decrease endurance performance in athletes due to associated muscle and/or liver glycogen depletion. Currently, no scientific evidence is available to support an ergogenic effect of capsaicin supplementation. Additional research is merited.

Ginkgo biloba. The Chinese Ginkgo tree is the world's most ancient extant, originating two hundred million years ago, and is the source for Ginkgo biloba leaf extract. Ginkgo biloba is believed to exert its mode of action when its active ingredients, the flavonoids and terpenoids, work in concert ${ }^{10}$. One of the tissue level effects is stimulated release of endothelium-derived relaxing factor, which may enhance muscle tissue blood flow through improved microcirculation ${ }^{11}$. Such an effect could improve aerobic endurance by enhancing muscle tissue oxidation.

Most Ginkgo biloba supplementation research has been conducted in the elderly, primarily for its vasoregulatory and cognition-enhancing effects. A meta-analysis ${ }^{12}$ and several subsequent studies ${ }^{13,14}$ have shown that Ginkgo biloba improves exercise performance, as evaluated by walking distance, in patients with peripheral arterial disease (PAD). However, although Ginkgo biloba supplementation may improve exercise endurance in patients with $\mathrm{PAD}$, there is no evidence that similar effects occur in healthy young or older athletes.

Ginseng. Ginseng is one of the most popular herbal dietary supplements worldwide. Sales in the United States have been reported to be over $\$ 300$ million annually ${ }^{15}$. Ginseng consists of several species belonging to the plant family Araliaceae. The two major forms are Chinese, Korean or Asian ginseng which belong to the genus Panax, and Siberian or Russian ginseng which belongs to the genus Eleutherococcus ${ }^{16}$. The biologically active constituents in Panax ginseng are a complex mixture of triterpene saponins known as ginsenosides. Siberian, or Russian, ginseng consists of the dried roots and rhizome of Eleutherococcus senticosis, and contains phenolics, polysaccharides, and eleutherosides. In China, Eleutherococcus senticosus is known as wujiaseng or Ciwujia, and the proposed active ingredients are ciwujianosides ${ }^{17}$.

The ergogenic effect of ginseng is attributed to the ginsenosides, eleutherosides, and ciwujianosides. Ginseng is theorized to influence the hypothalamicpituitary-adrenal cortex axis, possibly mitigating the catabolic effects of the stress hormone cortisol. Given these theorized anti-stress effects, one theory of ginseng supplementation is to enhance sports performance by allowing athletes to train more intensely or to induce an antifatiguing effect and increase stamina during competition. Other theories have been proposed to explain the potential ergogenic effect on aerobic endurance capacity, including favorable metabolic, hematologic, and cardiovascular functions. Given these postulates, much of the research involving the ergogenicity of ginseng supplementation has focused on cardiovascular or aerobic endurance performance, with some emphasis on psychomotor performance ${ }^{18}$.

Earlier research findings relative to the effect of ginseng on endurance performance are equivocal. For example, one reviewer ${ }^{19}$ indicated that controlled studies of Asian ginseng found improvement in exercise performance with use of standardized extracts, long duration of supplementation, large numbers of subjects, and elderly subjects. However, most earlier studies reporting positive ergogenic effects have been associated with improper research methodology, including no control or placebo group, 
no double-blind protocol, no randomization of order of treatment, no statistical analysis, or the use of nonstandardized commercial ginseng preparations containing other potential ergogenic substances $20,15,18$. Several recent studies have reported ergogenic effects of Panax ginseng. Liang and others ${ }^{21}$ reported that Panax ginseng supplementation ( $1.35 \mathrm{~g}$ /day for 30 days) significantly increased cycle ergometer endurance time in untrained adults. Also using untrained adults, Kim and others ${ }^{22}$ found that eight weeks supplementation with Panax ginseng extract (6 $\mathrm{g} /$ day) enhanced performance in treadmill running time which, based on serum levels of antioxidant enzymes, was attributed to decreased oxidative stress. However, this one-group study involved a control pre-test followed by a post-test after the eight-week supplementation period; no placebo was utilized.

The vast majority of well-controlled studies have reported no significant effect of either Panax ginseng, eleutherococcus senticosus Maxim L, Ciwujia, or a standardized ginseng extract on cardiovascular, metabolic, or psychologic responses to either submaximal or maximal exercise performance, or on maximal or supramaximal performance capacity $23,17,24,25,26$. Several recent extensive reviews of well-designed studies have concluded that there is an absence of compelling research evidence regarding the efficacy of ginseng use to improve physical performance in humans $15,16,18$, with one of the reviews focusing solely on eleutherococcus senticosus ${ }^{28}$. Well-controlled studies and detailed reviews indicate that ginseng in its various forms does not enhance exercise or sport performance.

Kava kava (kava) and St. Johns wort. Kava kava, or kava, is the peeled and dried root of Piper methysticum G. Forster, a centuries-old South Pacific herb used as a ritual beverage for its relaxing or calming properties. Kava root contains kava lactones (kava pyrones). The neuropharmacologic effects of kava include analgesia, sedation, and skeletal muscle relaxation, but not central nervous system depression. The mechanism is not clear, but blockage of the GABA or norepinephrine neuroreceptors may be involved ${ }^{5,29}$. St. John's wort (SJW) consists of the dried parts of Hypericum perforatum. SJW contains many phytochemicals, including flavonoids, phenolic acids, sterols, tannins, two naphthodianthrones (hypericin and pseudohypericin), and a phloroglucinol derivative (hyperforin). SJW is used therapeutically as an antidepressant. Hyperforin is thought to be the primary active ingredient in antidepressant activity, but hypericin and pseudohypericin may also be important. All are thought to help maintain optimal brain neurotransmitter levels including serotonin, norepinephrine and dopamine ${ }^{5,29}$.

Kava is theorized to reduce excess anxiety and/or hand tremor that may disrupt performance in many sports, such as archery and pistol shooting. Kava has been marketed for its antidepressant or anti-anxiety effects, a possible alternative to prohibited or potentially risky ergogenic drugs in such sports, such as alcohol and beta blockers ${ }^{30}$. Comparable to kava, SJW may be theorized to reduce anxiety and hand tremor in some athletes ${ }^{31}$. Additionally, as serotonin is involved in appetite control, SJW is theorized to help induce weight loss, which could confer a mechanical advantage to some athletes.

A recent meta-analysis has suggested that kava extract may be effective for reduction of anxiety symptoms ${ }^{32}$, while reviews and meta-analyses of research with St. John's wort conclude its effects on treatment of depression are inconsistent and confusing, some showing benefits comparable to standard antidepressant drugs while others noting minimal beneficial effects ${ }^{33-35}$. Unfortunately, however, no research evaluating the potential ergogenic effect of kava or SJW supplementation on exercise or sport performance has been uncovered. Moreover, no data are available supporting SJW as a means of promoting leanness in athletes ${ }^{18}$.

Gamma oryzanol and Tribulus terrestris. Gammaoryzanol, a mixture of ferulic acid esters of sterol and triterpene alcohols occurs in rice (Oryza stavia) bran oil at a level of 1-2 percent. Tribulus terrestris, commonly known as puncture vine, is an herbal preparation that has been used medicinally as a diuretic as well as treatment for hypertension and hypercholesterolemia, and has been used for centuries in Europe as treatment for impotence. The purported active ingredients are saponins and protodyosin. As ergogenic aids, both gamma oryzanol and Tribulus terrestris are used in the belief that they may elicit anabolic effects via increased testosterone production ${ }^{36,37,18}$.

Although limited, the available research does not support an ergogenic effect of either gamma-oryzanol or Tribulus terrestris supplementation in humans. Compared to a placebo group, weight-trained males who consumed $500 \mathrm{mg} /$ day of gamma-oryzanol for 9 weeks of resistance exercise training experienced no significant differences in circulating concentrations of hormones (testosterone, human growth hormone, 
insulin), nor was there any improvement in muscular power or strength ${ }^{38}$. Neychev and Mitev ${ }^{39}$ found that consumption of either 10 or $20 \mathrm{mg} / \mathrm{kg}$ body weight of Tribulus terrestris extract daily for four weeks had no effect on serum testosterone or androstenedione. Also, in a double-blind, placebo-controlled study, Tribulus terrestris supplementation exerted no effect on body weight, body composition, maximal strength or muscular endurance in resistance-trained males during training; plasma testosterone levels were not measured ${ }^{36}$.

\section{OTHER PURPORTED HERBAL ERGOGENICS}

Several other herbals have been studied for their purported ergogenic potential. Cordyceps sinensis is theorized to have favorable effects on the heart and circulation to improve oxidative capacity and endurance performance. Natural Cordyceps sinensis is rare, but a synthetic version is available; one version is CordyMax Cs-4. Parcell and others ${ }^{40}$ reported that 5 weeks of CordyMax Cs-4 supplementation had no effect on aerobic capacity or endurance exercise performance in endurance-trained male cyclists. Rhodiola rosea has been theorized to enhance endurance performance through a stimulating effect. In a preliminary study, De Bock and others ${ }^{41}$ found that an acute dose (200 milligrams) of Rhodiola rosea improved time to exhaustion by 3 percent on a cycle ergometer, but there was no significant effect following four weeks of supplementation with 200 milligrams daily. There was no effect on maximal strength or various measures of reaction time or movement time. Using combinations of such herbals have also been shown to have no ergogenic effect. Colson and others ${ }^{42}$ and Earnest and others ${ }^{43}$ evaluated the ergogenic effects of a Cordyceps sinensis and Rhodiola rosea-based supplement and reported no significant effects on oxygen dynamics, various physiological measures, or cycling time to exhaustion. Cytoseira canariensis has been marketed to increase muscle mass and decrease body fat by inhibiting myostatin, a growth and differentiation factor whose role is to inhibit (not promote) the growth of muscles. However, Willoughby ${ }^{44}$ reported that 1,200 milligrams/day of Cystoseira canariensis supplementation during 12 weeks of resistance training had no effect on serum myostatin levels, not did it have any effect on muscle mass, muscle strength, or body fat. Pittler and Ernst 45 reviewed the research on numerous dietary supplements marketed for weight loss, including various herbals, and found that none (with the possible exception of ephedra) have shown evidence beyond a reasonable doubt that they are effective for reducing body weight.

\section{SUMMARY}

Numerous herbal supplements are marketed as ergogenic aids for athletes. Although ginseng has received some considerable research attention, there is a dearth of well-controlled research evaluating the efficacy of purported herbal ergogenics on human exercise or sport performance ${ }^{46,18}$. Much of our knowledge concerning the efficacy of these herbal ergogenics is anecdotal in nature, and much of the earlier research that is available suffers from methodological problems such as poor research design and use of a variety of substances where the purity and content are often suspect. Future research efforts require careful attention to experimental design, product purity, standardized dosages, subject compliance, and statistical power.

From a health viewpoint, many contemporary herbal medicines have survived for centuries because they are believed to have therapeutic medicinal (although not ergogenic) value applicable to physically active individuals. However, a recent survey by the Consumers Union ${ }^{47}$ suggests most well-known, heavily promoted herbal treatments may not be very effective. Moreover, many may not be safe and may have some serious side effects, particularly when used in excessive amounts or when combined with other herbs or drugs ${ }^{48,46,49}$. Commercially-available herbal preparations also may contain proscribed pharmacological agents, such as anabolic steroids, which may lead to positive doping tests ${ }^{50}$. Thus, physically active individuals who desire to use herbal supplements should consult appropriate healthcare professionals beforehand because not all herbal supplements are safe or permitted for use in sport. Safety information regarding herbal and other dietary supplements may be found at www.fda.gov/medwatch.

\section{REFERENCES}

1. Winslow, L. C., and Kroll, D. J. Herbs as medicines. Archives of Internal Medicine v.158, p. 2192-2199, 1998.

2. Sengupta, S., Toh, S., Sellers, L., et al. Modulating angiogenesis: The yin and yang in ginseng. Circulation 110:1219-25, 2004.

3. Ervin, R., Wright, J., and Reed-Gillette, D. Prevalence of leading types of dietary supplements used in the Third National Health 
and Nutrition Examination Survey, 1988-194. Advance Data 9 (349):1-7, 2004.

4. Herbold, N., Visconti, B., Frates, S, and Bandini, L. Traditional and nontraditional supplement use by collegiate female varsity athletes. International Journal of Sport Nutrition and Exercise Metabolism 14:586-93, 2004.

5. Blumenthal, M., Goldberg, A., and Brinckmann, J. Herbal Medicine. Newton, MA: Integrative Medicine Communications, 2000.

6. World Health Organization. WHO Monographs on Selected Medicinal Plants. Geneva: World Health Organization, 1999.

7. Tyler, V. E. The Honest Herbal: A Sensible Guide to the Use of Herbs and Related Remedies New York: Pharmaceutical Products Press, 1993.

8. Lim, K., et al. Dietary red pepper ingestion increases carbohydrate oxidation at rest and during exercise in runners. Medicine and Science in Sports and Exercise v. 29, p. 355-361, 1997.

9. Glickman-Weiss, E. L. et al. Does capsaicin affect physiologic and thermal responses of males during immersion in 22 degrees C? Aviation Space and Environmental Medicine v. 69, p. 1095-1099, 1998.

10. Curtis-Prior, P. et al. Therapeutic value of Ginkgo biloba in reducing symptoms of decline in mental function. Journal of Pharmacy and Pharmacology. v. 51, p. 535-541, 1999.

11. Cott, J. NCDEU update: Natural product formulations available in Europe for psychotropic indications. Psychopharmacology Bulletin. v. 31, p. 745-51, 1995.

12. Schneider, B. Ginkgo biloba extract in peripheral arterial diseases: Meta-analysis of controlled clinical studies. Arzneimittelforschung v. 42, p. 428-436, 1992.

13. Blume, J. et al. Placebo-controlled double-blind study of the effectiveness of Ginkgo biloba special extract EGb 761 in trained patients with intermittent claudication. Vasa. v. 25, p. 265-274, 1996.

14. Peters, H., Kieser, M., and Holscher, U. Demonstration of the efficacy of ginkgo biloba special extract EGb 761 on intermittent claudication: A placebo-controlled, double-blind multicenter trial. Vasa. v. 27, p. 106-110, 1998.

15. Bahrke, M. S., and Morgan, W. P. Evaluation of the ergogenic properties of ginseng: An update. Sports Medicine. v. 29, p. 113$133,2000$.

16. Vogler, B. K., Pittler, M. H., and Ernst, E. The efficacy of ginseng: A systematic review of randomized clinical trials. European Journal of Clinical Pharmacology. V. 55, p. 567-575, 1999.

17. Cheuvront, S. N., et al. Effect of ENDUROX ${ }^{\mathrm{TM}}$ on metabolic responses to submaximal exercise. International Journal of Sport Nutrition. v.9, p. 434-442, 1999.

18. Williams, M. H., and Branch, J. D. Herbals as ergogenic aids. In Performance-Enhancing Substances in Sport and Exercise. M. Bahrke and C. Yesalis (Eds.). Champaign, IL: Human Kinetics, 2002.

19. Bucci, L. Selected herbals and human exercise performance. American Journal of Clinical Nutrition. v. 72, p. 624S-636S, 2000

20. Bahrke, M. S., and Morgan, W. P. Evaluation of the ergogenic properties of ginseng. Sports Medicine. v. 18, p. 229-248, 1994.

21 Liang, C., Podolka, T., Chuang, W. Panax notoginseng supplementation enhances physical performance during endurance exercise. Journal of Strength and Conditioning Research 19:108-14, 2005.

22. Kim, S., Park, K., Chang, M., and Sung, J. Effects of Panax ginseng extract on exercise-induced oxidative stress. Journal of Sports Medicine and Physical Fitness 45:178-82, 2005.

23. Allen, J. D., et al. Ginseng supplementation does not enhance healthy young adults' peak aerobic exercise performance. Journal of the American College of Nutrition. v. 17, p. 462-6, 1998.

24. Dowling, E. A. et al. Effect of Eleutherococcus senticosus on submaximal and maximal exercise performance. Medicine and Science in Sports and Exercise. v. 28, p. 482-489, 1996.

25. Engels, H. et al. 2001. Effects of ginseng supplementation on supramaximal exercise performance and short-term recovery. Journal of Strength and Conditioning Research. v.15, p. 290-295.

26. Engels, H-J., and Wirth, J. C. No ergogenic effects of ginseng (Panax ginseng C. A. Meyer) during graded maximal aerobic exercise. Journal of the American Dietetic Association. v. 97, p. 1110-1115, 1997.

27. Morris, A. C. et al. No ergogenic effect of ginseng ingestion. International Journal of Sport Nutrition. v. 6, p. 263-271, 1996.

28. Goulet, E., and Dionne, I. 2005. Assessment of the effects of Eleutherococcus Senticosus on endurance performance. International Journal of Sport Nutrition and Exercise Metabolism 15:75-83.

29. Singh, Y. Potential for interaction of kava and St. John's wort with drugs. Journal of Ethnopharmacology 100 (1-2):108-13, 2005.

30. Williams, M. H. Alcohol, marijuana, and beta blockers. In Ergogenics: Enhancement of Performance in Exercise and Sport, Eds. D. R. Lamb and M. H. Williams. Dubuque: WCB/Brown \& Benchmark, 1991.

31. Anderson, O. St. John's wort: A nice mood-lifter for rueful runners? Running Research News. V. 14, n. 3, p. 1, 7-10, 1998.

32. Pittler, M. H., and Ernst, E. Efficacy of kava extract for treating anxiety: Systematic review and meta-analysis. Journal of Clinical Psychopharmacology. v. 20, p. 84-89, 2000.

33. Gaster, B., and Holroyd, J. St. John's wort for depression: A systematic review. Archives of Internal Medicine. v. 160, p. 152-156, 2000.

34. Linde, K., Mulrow, C., Berner, M., and Egger, M. St. John's wort for depression. Cochrane Database of Systematic Reviews 18 (2):CD000448, 2005.

35. Werneke, U., Horn, O., and Taylor, D. How effective is St John's wort? The evidence revisited. Journal of Clinical Psychiatry 65:611-17, 2004.

36. Antonio, J., et al. The effects of Tribulus Terrestris on body composition and exercise performance in resistance-trained males. International Journal of Sport Nutrition and Exercise Metabolism. v. 10, p. 208-215, 2000.

37. Wheeler, K. B., and Garleb, K. A. Gamma oryzanol-plant sterol supplementation: Metabolic, endocrine, and physiologic effects. International Journal of Sport Nutrition. v. 1, p. 170-177, 1991.

38. Fry, A. C. et al. The effects of gamma-oryzanol supplementation during resistance exercise training. International Journal of Sport Nutrition. v. 7, p. 318-329, 1997.

39. Neychev, V., and Mitev, V. The aphrodisiac herb Tribulus terrestris does not influence the androgen production in young men. Journal of Ethnopharmacology 101 (1-3):319-23, 2005.

40. Parcell, A., Smith, J., Schulthies, S., et al. Cordyceps Sinensis (CordyMax Cs-4) supplementation does not improve endurance 
exercise performance. International Journal of Sport Nutrition and Exercise Metabolism 14:236-42, 2004.

41. De Bock, K., Eijnde , B., Ramaekers, M., and Hespel, P. Acute Rhodiola rosea intake can improve endurance exercise performance. International Journal of Sport Nutrition and Exercise Metabolism 14:298-307, 2004.

42. Colson, S., Wyatt, F., Johnston, D., et al. Cordyceps sinensis and Rhodiola rosea-based supplementation in male cyclists and its effect on muscle tissue oxygen saturation. Journal of Strength and Conditioning Research 19:358-63, 2005.

43. Earnest, C., Morss, G., Wyatt, F., et al. Effects of a commercial herbal-based formula on exercise performance in cyclists. Medicine \& Science in Sports \& Exercise 36:504-09, 2004.

44. Willoughby, D. Effects of an alleged myostatin-binding supplement and heavy resistance training on serum myostatin, muscle strength and mass, and body composition. International Journal of Sport Nutrition and Exercise Metabolism 14:461-72, 2004.

45. Pittler, M., and Ernst, E. Dietary supplements for body-weight reduction: A systematic review. American Journal of Clinical Nutrition 79:529-36, 2004.

46. Kundrat, S. 2005. Herbs and athletes. Sports Science Exchange 18 (1):1-6.

47. Consumers Union. Which alternative treatments work? Consumer Reports 70 (8):39-43, 2005.

48. Ernst, E. Risks of herbal medicinal products. Pharmacoepidemiology and Drug Safety 13:767-71, 2004.

49. Pittler, M., Schmidt, K., Ernst, E. Adverse effects of herbal food supplements for body weight reduction. Obesity Reviews 6:93$111,2005$.

50. Maughan, R., King, D., and Lea, T. Dietary supplements. Journal of Sports Sciences 22:95-103, 2004. 